University of Nebraska - Lincoln

DigitalCommons@University of Nebraska - Lincoln

Faculty Publications, Department of Physics and Astronomy

Research Papers in Physics and Astronomy

2019

\title{
Magnetoelectric control of topological phases in graphene
}

\author{
Hiroyuki Takenaka \\ University of Nebraska - Lincoln, htakenaka2@unl.edu \\ Shane Sandhoefner \\ University of Nebraska - Lincoln, ssandhoefner3@unl.edu \\ Alexey Kovalev \\ University of Nebraska - Lincoln, alexey.kovalev@unl.edu \\ Evgeny Tsymbal \\ University of Nebraska-Lincoln, tsymbal@unl.edu
}

Follow this and additional works at: https://digitalcommons.unl.edu/physicsfacpub

Part of the Condensed Matter Physics Commons

Takenaka, Hiroyuki; Sandhoefner, Shane; Kovalev, Alexey; and Tsymbal, Evgeny, "Magnetoelectric control of topological phases in graphene" (2019). Faculty Publications, Department of Physics and Astronomy. 304.

https://digitalcommons.unl.edu/physicsfacpub/304

This Article is brought to you for free and open access by the Research Papers in Physics and Astronomy at DigitalCommons@University of Nebraska - Lincoln. It has been accepted for inclusion in Faculty Publications, Department of Physics and Astronomy by an authorized administrator of DigitalCommons@University of Nebraska - Lincoln. 


\title{
Magnetoelectric control of topological phases in graphene
}

\author{
Hiroyuki Takenaka, Shane Sandhoefner, Alexey A. Kovalev, and Evgeny Y. Tsymbal $\oplus^{\dagger}$ \\ Department of Physics and Astronomy and Nebraska Center for Materials and Nanoscience, \\ University of Nebraska, Lincoln, Nebraska 68588-0299, USA
}

(Received 14 July 2019; published 25 September 2019)

\begin{abstract}
Topological antiferromagnetic (AFM) spintronics is an emerging field of research, which involves the topological electronic states coupled to the AFM order parameter known as the Néel vector. The control of these states is envisioned through manipulation of the Néel vector by spin-orbit torques driven by electric currents. Here we propose a different approach favorable for low-power AFM spintronics, where the control of the topological states in a two-dimensional material, such as graphene, is performed via the proximity effect by the voltage induced switching of the Néel vector in an adjacent magnetoelectric AFM insulator, such as chromia. Mediated by the symmetry protected boundary magnetization and the induced Rashba-type spin-orbit coupling at the interface between graphene and chromia, the emergent topological phases in graphene can be controlled by the Néel vector. Using density functional theory and tight-binding Hamiltonian approaches, we model a graphene/ $\mathrm{Cr}_{2} \mathrm{O}_{3}$ (0001) interface and demonstrate nontrivial band gap openings in the graphene Dirac bands asymmetric between the $K$ and $K^{\prime}$ valleys. This gives rise to an unconventional quantum anomalous Hall effect (QAHE) with a quantized value of $2 e^{2} / h$ and an additional steplike feature at a value close to $e^{2} / 2 h$, and the emergence of the spin-polarized valley Hall effect (VHE). Furthermore, depending on the Néel vector orientation, we predict the appearance and transformation of different topological phases in graphene across the $180^{\circ} \mathrm{AFM}$ domain wall, involving the QAHE, the valley-polarized QAHE, and the quantum VHE, and the emergence of the chiral edge states along the domain wall. These topological properties are controlled by voltage through magnetoelectric switching of the AFM insulator with no need for spin-orbit torques.
\end{abstract}

DOI: 10.1103/PhysRevB.100.125156

\section{INTRODUCTION}

In the past decades, spintronics has been considered as a promising avenue to establish new frontiers in information technology by exploiting the spin degree of freedom [1]. Driven by this technological challenge, exploration of new spintronic phenomena has become one of the most active research topics in condensed matter physics. Recently, antiferromagnetic (AFM) spintronics has emerged as a subfield of spintronics, where the AFM order parameter known as the Néel vector was employed as the nonvolatile state variable [2-5]. Due to being robust against magnetic perturbations and exhibiting ultrafast dynamics, antiferromagnets can serve as promising functional materials for spintronic applications.

In parallel with these developments, there has been increasing interest in materials and structures where quantum effects are responsible for novel physical properties, revealing the important roles of symmetry, topology, and dimensionality [6]. Among such quantum materials are graphene [7], topological insulators [8], Dirac and Weyl semimetals [9], and beyond [10]. The unique spin-dependent electronic properties of these materials are envisioned to open new perspectives for spintronic applications [11-13]. Among them, topological AFM spintronics is especially interesting, involving the interplay between the topological electronic states and antiferromagnetism [14-17].

\footnotetext{
*htakenaka2@unl.edu

†tsymbal@unl.edu
}

The key ingredient of AFM spintronics, including its topological variant, is a possibility to control the Néel vector by an external stimulus. Achieving this functionality in antiferromagnets is not straightforward as in ferromagnets where the control of the magnetic order parameter, i.e., magnetization, can be realized by an applied magnetic field, spin transfer torque, or spin-orbit torque. Recently, it has been predicted that in antiferromagnets with two spin sublattices forming inversion partners, an electrical current can induce a nonequilibrium magnetic field, whose sign alternates between the spin sublattices [18]. Such a staggered magnetic field generates an alternating sign in spin-orbit torque, which can trigger Néel vector switching. This prediction has been realized experimentally for CuMnAs [19] and $\mathrm{Mn}_{2} \mathrm{Au}$ [20] antiferromagnets, thus demonstrating a viable route for AFM spintronics.

While this progress is impressive, it is recognized that very large electric currents are needed to generate the required spin-orbit torques $\left(>10^{6} \mathrm{~A} \mathrm{~cm}^{-2}\right)$. Such currents would inevitably produce significant energy dissipation unfavorable for the desired low-power spintronics. From this perspective, it is beneficial to have the ability of controlling the Néel vector purely by electric fields through an applied voltage. Such a control has been demonstrated using the magnetoelectric antiferromagnet $\mathrm{Cr}_{2} \mathrm{O}_{3}$ in the corundum structure (chromia) [21]. Due to the magnetoelectric nature of this material, by applying an electric field (in the presence of a finite magnetic bias), one can switch the Néel vector between two nonvolatile states. Utilizing this functionality in topological AFM spintronics 
(a)

(b)
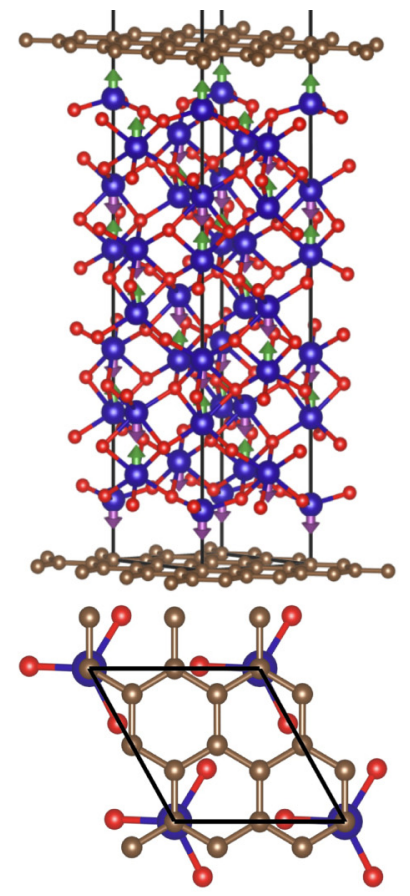

(c)

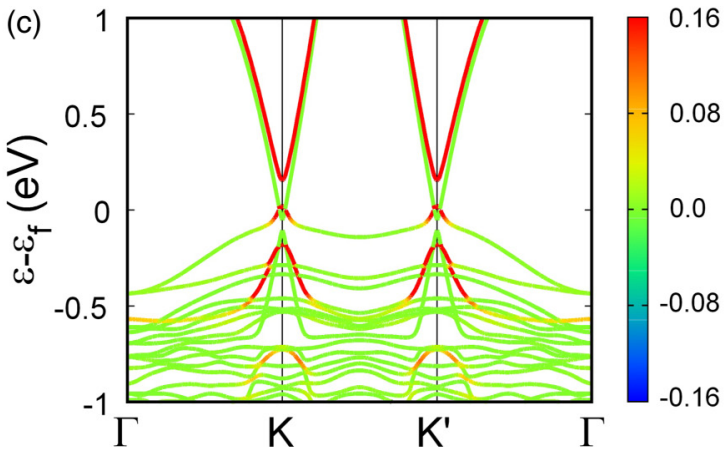

(d)

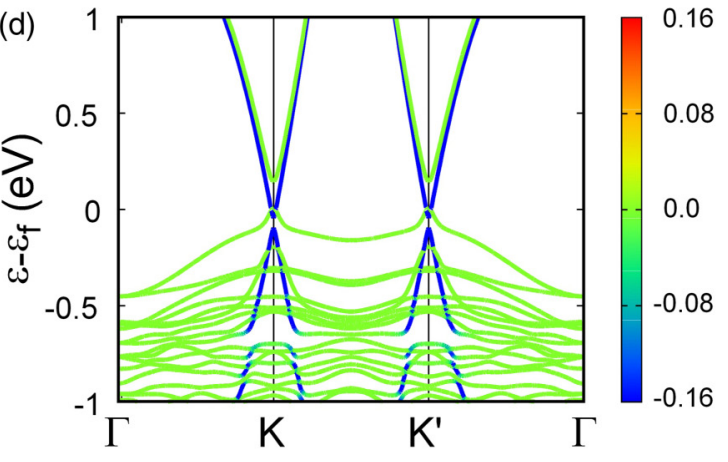

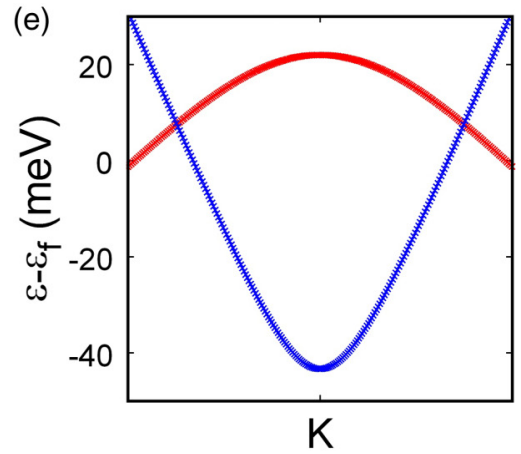

(f)

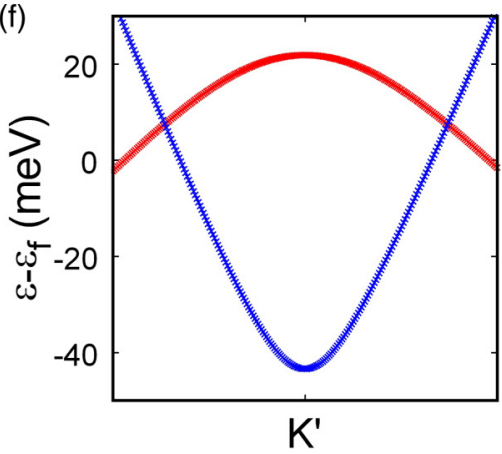

FIG. 1. Atomic and electronic structure of the graphene/ $\mathrm{Cr}_{2} \mathrm{O}_{3}(0001)$ interface without SOC. (a) The optimized atomic structure: side view. Blue, red, and gold balls indicate $\mathrm{Cr}, \mathrm{O}$, and $\mathrm{C}$ atoms, respectively. Color arrows denote up (green) and down (purple) spins in AFM chromia. (b) Top view of the atomic structure (a) showing graphene and surface $\mathrm{Cr}$ and subsurface O monolayers. (c), (d) Electronic band structures projected to the top graphene layer for spin-up (c) and spin-down (d) electrons. The color contrast reflects the strength of the carbon $p_{z}$ orbital contribution weighted with the $s_{z}$ spin contribution in arbitrary units. (e), (f) The spin-resolved bands originating from the graphene Dirac bands zoomed in near $K$ (e) and $K^{\prime}$ (f) points. The same color coding is used as in (c), (d).

would provide both ultralow power performance and ultrafast switching dynamics.

To this end, the voltage-switchable Néel vector in chromia could be exploited to manipulate quantum and topological properties of two-dimensional materials, such as graphene, via the proximity effect. The exchange coupling between chromia and graphene across the interface in a graphene/ $\mathrm{Cr}_{2} \mathrm{O}_{3}$ hybrid structure would be mediated by the boundary magnetization, which is an intrinsic property of all magnetoelectric antiferromagnets [22-24]. The boundary magnetization is firmly coupled to the bulk AFM order so that switching of the Néel vector leads to its reversal. Due to being insensitive to the interface roughness, the boundary magnetization can serve as a robust voltage-controlled parameter to operate topological properties of graphene. Experimental efforts along these lines have indicated the potential of the graphene $/ \mathrm{Cr}_{2} \mathrm{O}_{3}$ hybrid structure for realizing a magnetoelectric transistor [25].

The appearance of topological effects in graphene, such as the quantum anomalous Hall effect (QAHE) [26] and the quantum spin Hall effect (QSHE) [27] requires spin-orbit coupling (SOC). It is known, however, that the intrinsic SOC in pristine graphene is extremely weak $[28,29]$. Yet, a sizable SOC in graphene can be induced by the proximity effect at the interface between graphene and other materials such as transition metal dichalcogenides [30,31]. In addition to SOC, the proximity effect can also induce a sizable spin polarization in graphene when it is deposited on the surface of a magnetic insulator [32,33], which can lead to the QAHE [34].
All these observations indicate that the proximity effect at the $\mathrm{Cr}_{2} \mathrm{O}_{3}$ /graphene interface could produce SOC necessary for graphene to exhibit topological properties, which could be controlled by voltage through the boundary magnetization of chromia. Motivated by this idea, we use density functional theory (DFT) and model tight-binding approaches to explore spin- and orbital-dependent electronic and transport properties of the graphene/ $\mathrm{Cr}_{2} \mathrm{O}_{3}$ (0001) interface. We show that the presence of a sizable SOC and exchange splitting in graphene induced by the proximity of chromia leads to the QAHE which changes sign with reversal of the $\mathrm{Cr}_{2} \mathrm{O}_{3}$ Néel vector. The broken symmetry between the $A$ and $B$ sublattices in graphene at the $\mathrm{Cr}_{2} \mathrm{O}_{3}$ /graphene interface produces asymmetry between the $K$ and $K^{\prime}$ valleys, resulting in an unconventional QAHE and the emergence of the spin-polarized valley Hall effect. We predict the appearance and change of different topological phases in graphene across the $180^{\circ} \mathrm{AFM}$ domain wall and the emergence of the chiral edge states along the domain wall.

\section{RESULTS}

\section{A. DFT calculations}

We perform DFT calculations of the graphene/ $/ \mathrm{Cr}_{2} \mathrm{O}_{3}$ (0001) interface, as described in the Appendix for computational details. A $2 \times 2$ unit cell of graphene excellently matches to the $1 \times 1$ unit cell of the $\mathrm{Cr}_{2} \mathrm{O}_{3}$ (0001) surface with a lattice mismatch of just $0.83 \%$. By performing structural 

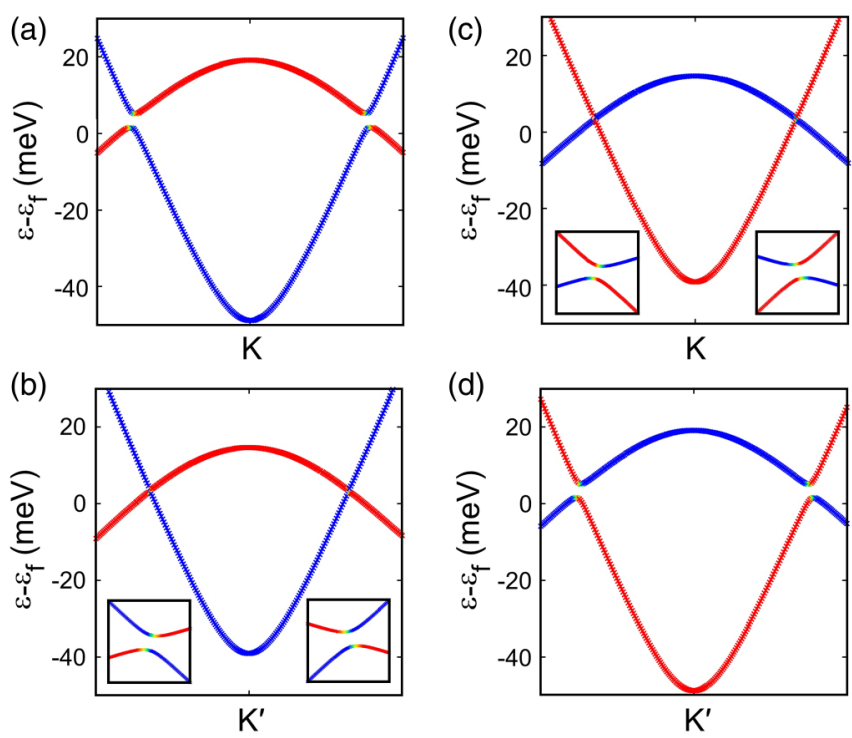

FIG. 2. The DFT-calculated band structures of the graphene/ $\mathrm{Cr}_{2} \mathrm{O}_{3}$ (0001) interface in the presence of SOC. (a), (b) The band structures around the $K$ (a) and $K^{\prime}$ (b) points. (c), (d) Same as (a), (b), respectively, but for the reversed Néel vector in chromia. Color contrast reflects the $s_{z}$ spin contribution in the same way as in Fig. 1. The insets in (b), (c) are the zoomed bands to reveal small band openings of $0.7 \mathrm{meV}$.

optimization (Supplemental Material [35]), we find the most energetically favorable atomic structure, which is shown in Figs. 1(a) and 1(b). In this interface structure, one $\mathrm{C}$ atom lies atop the surface $\mathrm{Cr}$ atom, the distance between the two being $2.63 \AA$. Such a large distance indicates the weak bonding between graphene and the substrate consistent with the previous DFT calculations [32,34].

The magnetic structure of bulk $\mathrm{Cr}_{2} \mathrm{O}_{3}$ represents a collinear antiferromagnetic configuration with $\mathrm{Cr}$ magnetic moments pointing along the (0001) direction [Fig. 1(a)]. The top surface $\mathrm{Cr}$ monolayer has parallel-aligned magnetic moments of $m_{\mathrm{Cr}}=2.8 \mu_{\mathrm{B}}$, representing the boundary (surface) magnetization [23]. Reversal of the Néel vector in bulk chromia (which can be achieved by voltage) leads to the reversal of this surface magnetization.

We find that there is a sizable exchange splitting of the spin bands in graphene induced by the proximity of the surface magnetization of chromia. Figures 1(c) and 1(d) show the band structure of the graphene/ $\mathrm{Cr}_{2} \mathrm{O}_{3}$ (0001) interface calculated without SOC in the vicinity of the Dirac point of pristine graphene. Here the bands are projected to the $p_{z}$ orbitals of the top graphene layer and their up- [Fig. 1(c)] and down- [Fig. 1(d)] spin weights are shown in color. It is seen that the spin bands are split by the induced exchange interaction. It is also seen that there is a splitting between bands of the same spin which is due to a staggered sublattice potential discussed below. Figures 1(e) and 1(f) zoom in on the spin-split bands originating from the graphene Dirac bands near the $K$ and $K^{\prime}$ points, respectively. These figures reveal that the exchange splitting of the spin bands is about $60 \mathrm{meV}$, the band structures at the $K$ and $K^{\prime}$ points are identical, and there are no gap openings at the band crossing points in the absence of SOC.

The broken inversion symmetry at the graphene/ $\mathrm{Cr}_{2} \mathrm{O}_{3}$ interface gives rise to the Rashba-like SOC. The SOC mixes the up- and down-spin states and opens the gaps at the crossing points, as is evident from Figs. 2(a) and 2(b). We find that the band opening is about $3 \mathrm{meV}$ near the $K$ valley [Fig. 2(a)] and is about $0.7 \mathrm{meV}$ near the $K^{\prime}$ valley [Fig. 2(b)]. From comparison of Figs. 2(a) and 2(b), it is also notable that SOC reduces the effective spin splitting at the $K^{\prime}$ valley. This difference in the band structure at the $K$ and $K^{\prime}$ points is due to the variable bond length between $\mathrm{Cr}$ surface atoms and $\mathrm{C}$ atoms in the graphene $A$ and $B$ sublattices [Fig. 1(b)], resulting in the staggered potential, the staggered exchange interaction, and the staggered SOC, as described by our tight-binding model below.

Switching the Néel vector in chromia is equivalent to the time-reversal symmetry transformation. It is therefore expected that reversal of all the $\mathrm{Cr}$ magnetic moments in $\mathrm{Cr}_{2} \mathrm{O}_{3}$ would lead to swapping the band structures between the $K$ and $K^{\prime}$ points with simultaneous reversal of the spin character of the bands. This is exactly what we find by performing self-consistent DFT calculations in the presence of SOC for the graphene/ $\mathrm{Cr}_{2} \mathrm{O}_{3}$ interface. From comparison of Figs. 2(c) and 2(d), and Figs. 2(a) and 2(b), we see that switching the Néel vector in chromia transforms the band structures between the $K$ and $K^{\prime}$ points and at the same time reverses the spin character.

\section{B. Tight-binding model}

To provide more insight into the proximity effect on the electronic band structure of graphene and to analyze its quantum transport behavior, we build a model tight-binding Hamiltonian as follows [31,36-39]:

$$
\begin{aligned}
H= & -t \sum_{\langle i j\rangle \alpha} c_{A i \alpha}^{\dagger} c_{B j \alpha}+i t_{s o} \sum_{\langle i j\rangle \alpha \beta} c_{A i \alpha}^{\dagger} \hat{z} \cdot\left(\vec{\sigma} \times \hat{d}_{i j}\right) c_{B j \beta}-\lambda_{n l} \sum_{\langle i j\rangle \alpha \beta} c_{A i \alpha}^{\dagger}(\hat{m} \cdot \vec{\sigma}) c_{B j \beta}+U \sum_{\mu=A, B} \eta_{\mu} \sum_{i \alpha} c_{\mu i \alpha}^{\dagger} c_{\mu i \alpha} \\
& +\sum_{\mu=A, B} i \lambda_{s o, \mu} \sum_{\langle\langle i j\rangle) \alpha \beta} v_{i j} c_{\mu i \alpha}^{\dagger} \sigma_{z} c_{\mu j \beta}-J_{A} \sum_{i \alpha \beta} c_{A i \alpha}^{\dagger}(\hat{m} \cdot \vec{\sigma}) c_{A i \beta}+J_{B} \sum_{i \alpha \beta} c_{B i \alpha}^{\dagger}(\hat{m} \cdot \vec{\sigma}) c_{B i \beta}+X \sum_{\mu=A, B} \sum_{i \alpha} c_{\mu i \alpha}^{\dagger} c_{\mu i \alpha},
\end{aligned}
$$

where $c_{A i \alpha}^{\dagger}$ and $c_{A i \alpha}$ are the creation and annihilation operators for site $i$ on sublattice $A$ with spin $\alpha$. The first term is the conventional tight-binding Hamiltonian for pristine graphene with hopping parameter $t$. The second term is the 

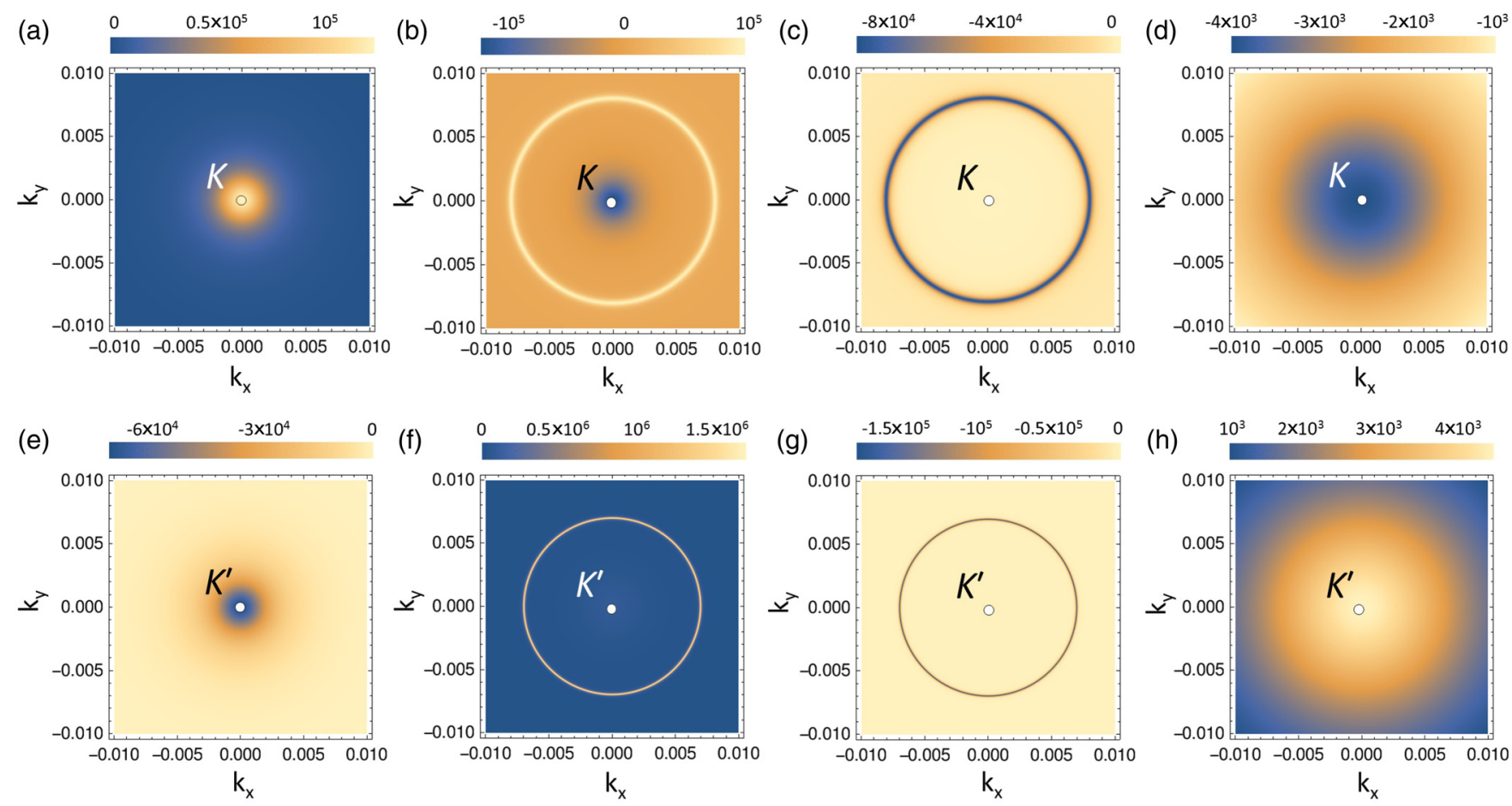

FIG. 3. Color contour plots of the Berry curvature projected on the $k_{x}-k_{y}$ plane. (a)-(d) The Berry curvature for the four bands shown in Supplemental Fig. S3(a) [35] around the $K$ point. (e)-(h) The Berry curvature for the four bands shown in Supplemental Fig. S3(b) [35] around the $K^{\prime}$ point. The bands are ordered from low to high energy. The origin of the $x$ and $y$ axes is at the $K$ point in (a)-(d) and at the $K^{\prime}$ point in (e)-(h). Color bars quantify the Berry curvature.

Rashba SOC, which includes parameter $t_{\mathrm{so}}$, Pauli matrix vector $\vec{\sigma}=\left(\sigma_{x}, \sigma_{y}, \sigma_{z}\right)$, and the unit vector $\hat{d}_{i j}$ from site $j$ to $i$. The third term represents the nonlocal exchange interaction involving the spin-dependent hopping with $\hat{m}=$ $(\sin \theta \cos \varphi, \sin \theta \sin \varphi, \cos \theta)$ being the unit vector of the surface magnetization. This term affects the slope of band curvatures by strength $\lambda_{n l}$. For simplicity, we assume $\varphi=0$, but note that varying $\varphi$ only causes small changes in the band structure and does not affect our main results. The remaining terms describe the site-dependent interactions. The fourth term describes the staggered sublattice potential $\left(\eta_{A}=1\right.$ and $\eta_{B}=-1$ ) of strength $U$. The staggered potential opens the band gaps and rounds the bands near the Dirac points, but does not introduce differences in the band structure near the $K$ and $K^{\prime}$ valleys. The fifth term describes the staggered SOC of amplitude $\lambda_{\text {so }}$, which involves the second-nearest neighbor hopping and has $v_{i j}=1(-1)$ for clockwise (counterclockwise) hopping from site $j$ to $i$. The band openings in question are strongly influenced by the staggered SOC. The next two terms correspond to the exchange interactions of strengths $J_{A}$ and $J_{B}$, which are assumed to be different on the $A$ and $B$ sublattices. The last term describes an overall energy shift. The summation over $\langle i j\rangle$ and $\langle\langle i j\rangle\rangle$ in Eq. (1) runs over all the nearest and next-nearest neighbor sites, respectively. We note that although the model Hamiltonian contains many terms, all the terms appear to be necessary to quantitatively reproduce the DFT calculated band structures (Supplemental Fig. S3 [35]). Details of the fitting procedure and the resulting fitting parameters are given in the Supplemental Material [35].

\section{Berry curvature}

Next, using Hamiltonian (1) with the fitted parameters, we analyze the QAHE, which is determined by the Berry curvature [9]:

$$
\Omega^{n}(\vec{k})=-\sum_{n^{\prime} \neq n} \frac{2 \operatorname{Im}\left\langle\Psi_{n \vec{k}}\left|v_{x}\right| \Psi_{n^{\prime} \vec{k}}\left\langle\Psi_{n^{\prime} \vec{k}}\left|v_{y}\right| \Psi_{n \vec{k}}\right\rangle\right.}{\left(\varepsilon_{n^{\prime} \vec{k}}-\varepsilon_{n \vec{k}}\right)^{2}} .
$$

Here $n$ is the band index, $v_{x, y}=\partial H / \partial k_{x, y}$ is the velocity operator, and $\varepsilon_{n k}$ and $\Psi_{n k}$ are eigenvalues and eigenfunctions of the Hamiltonian at a given $k$ point within the Brillouin zone. For simplicity, we omit $x$ and $y$ indices in the notation for $\Omega^{n}(\vec{k})$ as well as for the anomalous Hall conductance (AHC), $\sigma$, below. In Fig. 3, we show the calculated Berry curvature around the $K$ [Figs. 3(a)-3(d)] and $K^{\prime}$ [Figs. 3(e)-3(h)] valleys for each of the four bands displayed in Supplemental Figs. S3(a) and S3(b), respectively [35]. It is seen that the Berry curvature becomes very large on the circles around the $K$ and $K^{\prime}$ points. The radii of these circles match the $k$ values at which the band openings appear due to SOC [Figs. 2(a) and 2(b)]. The enhancement of $\Omega^{n}(\vec{k})$ at the $K$ and $K^{\prime}$ points, especially pronounced for the lowest [Figs. 3(a) and 3(e)] and highest [Figs. 3(d) and 3(h)] energy bands, reflects their extrema at these points (Supplemental Figs. S3(a) and S3(b) [35]) resulting from the band opening produced by the staggered sublattice potential $U$. 

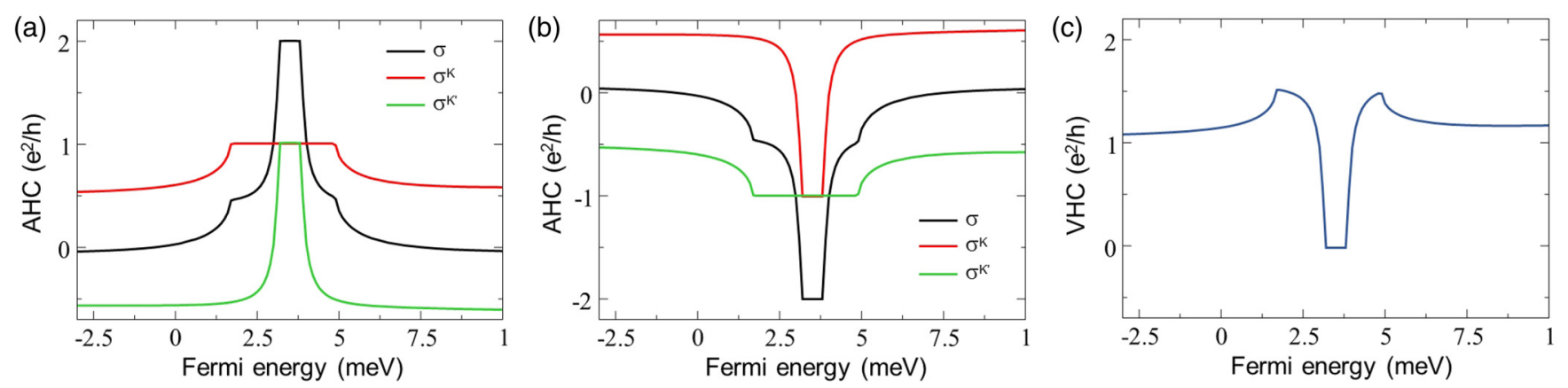

FIG. 4. The calculated anomalous Hall conductance (AHC) in graphene as a function of the Fermi energy $\varepsilon_{f}$ for the Néel vector in chromia pointing up (a) and down (b). $\sigma$ is the total AHC, and $\sigma^{K}$ and $\sigma^{K^{\prime}}$ are the partial contributions arising from the $K$ and $K^{\prime}$ valleys, respectively. (c) Valley Hall conductance (VHC), $\sigma_{v}=\sigma^{K}-\sigma^{K^{\prime}}$, as a function of $\varepsilon_{f}$. The results are obtained using Hamiltonian (1) with the parameters fitted to the DFT bands.

\section{Anomalous and valley Hall conductance}

The AHC is determined by the Berry curvature, as follows [9]:

$$
\sigma=\frac{e^{2}}{h} \frac{1}{2 \pi} \sum_{n} \int_{B Z} f_{n} \Omega^{n}(\vec{k}) d^{2} k,
$$

where $e$ is elementary charge, $h$ is Planck's constant, and $f_{n}$ is the Fermi-Dirac distribution function. Figure 4(a) (black line) shows the calculated AHC, $\sigma$, as a function of the Fermi energy, $\varepsilon_{f}$. As expected, the AHC acquires the value of $\sigma=$ $2 e^{2} / h$ when $\varepsilon_{f}$ lies within the energy region where there is a global energy gap in the system (i.e., where the band openings at the $K$ and $K^{\prime}$ points overlap). When $\varepsilon_{f}$ lies far from this gap, the AHC tends to zero due to the cancellation of the contributions from the $K$ and $K^{\prime}$ valleys. It is notable that in the vicinity of $\varepsilon_{f}=2 \mathrm{meV}$ and $\varepsilon_{f}=5 \mathrm{meV}$, i.e., above or below the smaller band gap, the AHC exhibits an unconventional two-step-like feature associated with quantized conductance at $e^{2} / h$ for one valley but not for the other. This feature in the AHC appears due to different band gaps at the $K$ and $K^{\prime}$ valleys. This is evident from Fig. 4(a), where we plot partial contributions to $\sigma$ arising from the $K$ and $K^{\prime}$ valleys, $\sigma^{K}$ and $\sigma^{K^{\prime}}$ [red and green lines in Fig. 4(a)], by integrating the Berry curvature over the respective $k$-space regions. Each of $\sigma^{K}$ and $\sigma^{K^{\prime}}$ has an exact quantized value of $e^{2} / h$ when $\varepsilon_{f}$ falls into the associated band gap. However, when $\varepsilon_{f}$ lies within the wider band of the $K$ valley, but above or below the band gap of the $K^{\prime}$ valley, the contribution from the latter is $\sigma^{K}=e^{2} / h$, while the contribution from the former, $\sigma^{K^{\prime}}$, drops down to about $-e^{2} / 2 h$ so that within this energy window $\sigma$ appears to be close to a value of $e^{2} / 2 h$. The $\sigma^{K^{\prime}}$ value of $-e^{2} / 2 h$ results from the integration of the Berry curvature of the lowest energy band around the $K^{\prime}$ valley [Fig. 3(e)]. Thus, the AHC exhibits the unconventional two-step-like behavior due to the different band openings at the $K$ and $K^{\prime}$ valleys.

Switching the Néel vector in chromia is equivalent to the time-reversal symmetry operation. Since $\Omega^{n}(\vec{k})$ is odd with respect to time-reversal symmetry, i.e., $\Omega^{n}(-\vec{k},-\vec{s})=$ $-\Omega^{n}(\vec{k}, \vec{s})$, where $\vec{s}$ is the spin, it is expected that with reversal of the Néel vector $\vec{L}, \sigma$ will change sign and the partial contributions will transform as $\sigma^{K}(-\vec{L})=-\sigma^{K^{\prime}}(\vec{L})$ and $\sigma^{K^{\prime}}(-\vec{L})=-\sigma^{K}(\vec{L})$. This is exactly what we find from the calculation shown in Fig. 4(b), where reversal of the Néel vector in the Hamiltonian (1) was modeled by changing the angle of the surface magnetization from $\theta=0^{\circ}$ to $\theta=180^{\circ}$. Since the Néel vector and the surface magnetization in chromia can be electrically switched, this result indicates the possibility of the voltage-controlled QAHE at low temperature. We note that a reversible AHE has been realized experimentally at room temperature, although using $\mathrm{Pt}$, rather than graphene, as an overlayer on chromia [40].

The asymmetry between the $K$ and $K^{\prime}$ valleys at the graphene/ $\mathrm{Cr}_{2} \mathrm{O}_{3}$ interface gives rise to the valley polarization [41]. The valley polarization appears due to different populations of the two valleys. It can be detected by measuring the longitudinal transport in graphene perpendicular to the AFM domain wall in chromia between two regions with opposite orientation of the Néel vector. Creation and annihilation of the AFM domain wall performs as a valley valve, filtering the valley-polarized carriers in graphene. If the chemical potential is engineered to be located within the wider band of the $K$ valley, but below or above the band gap of the $K^{\prime}$ valley, a perfect valley filtering is expected in the ballistic transport regime. In this case the longitudinal conductance is zero in the presence of the domain wall but nonzero in its absence.

The valley polarization gives rise to the valley Hall effect (VHE) [42]. The VHE can be quantified using a valley Hall conductance (VHC), which is defined as the difference in the AHC between the $K$ and $K^{\prime}$ valleys, i.e., $\sigma_{v}=\sigma^{K}-\sigma^{K^{\prime}}$. Figure 4(c) demonstrates that $\sigma_{v}$ is largest when the Fermi energy $\varepsilon_{f}$ lies within only one of the band gaps at either $K$ or $K^{\prime}$ valley (depending on magnetization orientation). $\sigma_{v}$ is zero if $\varepsilon_{f}$ lies within both gaps and is close to a quantized value of $e^{2} / h$ away from the gaps. The latter originates from the integration of the Berry curvature over the lowest energy band [Figs. 3(a) and 3(e)], contributing to the VHC $e^{2} / 2 h$ from the $K$ valley and $-e^{2} / 2 h$ from the $K^{\prime}$ valley. The VHC of $\sigma_{v} \approx e^{2} / h$ represents a spin-polarized version of the VHE [42], where the bands contributing to the transport are nearly fully spin polarized.

Contrary to $\sigma$, the sign of $\sigma_{v}$ does not depend on the Néel vector orientation (up or down). Due to this invariance, a pure valley current can be induced in graphene along the domain wall in $\mathrm{Cr}_{2} \mathrm{O}_{3}$. This effect can be realized and measured using a device structure in Fig. 5. Here a charge current is generated 


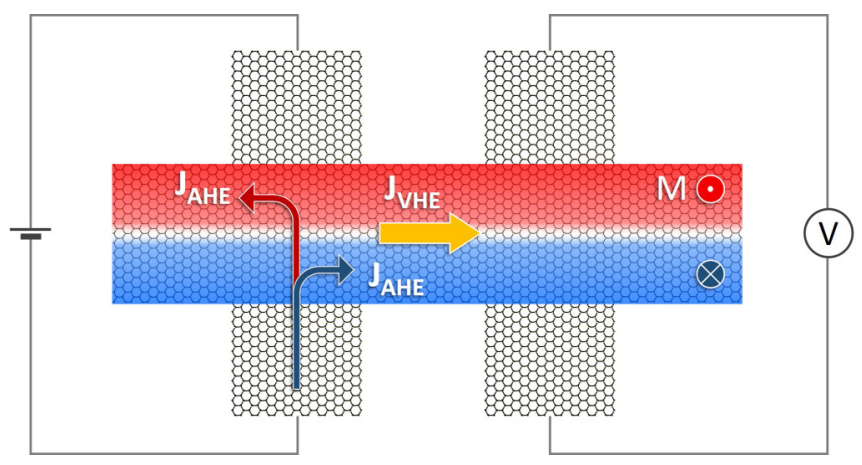

FIG. 5. Schematic set up for the detection of the valley Hall effect. Shaded regions represent two antiparallel-aligned domains of the surface magnetization in chromia. A charge current is generated along the left vertical graphene bar by a current source. It produces an anomalous Hall current, $J_{\mathrm{AHE}}$, which flows in the opposite directions above the two AFM domains of $\mathrm{Cr}_{2} \mathrm{O}_{3}$. The $\mathrm{AHC}$ has the opposite sign in the two domains and is thus canceled. On the contrary, a valley Hall current, $J_{\mathrm{VHE}}$, is not canceled and flows in graphene along the domain wall. Such a pure valley current induces a voltage drop between the top and bottom terminals of the right vertical graphene bar due to the inverse VHE.

along the left vertical graphene bar by a current source and produces an anomalous Hall current, $J_{\mathrm{AHE}}$, which flows in the opposite directions in the graphene layer regions above the two AFM domains of $\mathrm{Cr}_{2} \mathrm{O}_{3}$. If the domain wall is placed symmetrically (as in Fig. 5), the net anomalous Hall current is canceled. On the contrary, a valley Hall current, $J_{\mathrm{VHE}}$, is not canceled and flows in graphene along the domain wall. Such a pure valley current induces a voltage drop between the top and bottom terminals of the right vertical graphene bar in Fig. 5 due to the inverse VHE. This voltage drop can be detected using the nonlocal measurements, as was reported recently [43-45]. Note that careful setup engineering might be required due to possible shunting effect across the domain wall.

We note that the above scheme assumes that the Fermi level is located in the range of energies where both the AHC and VHC are nonzero. If the Fermi level is engineered to lie above or below the energy gaps, i.e., in the energy region where the $\mathrm{AHC}$ vanishes but the $\mathrm{VHC}$ does not, the $\mathrm{VHC}$ can also be measured using the proposed device scheme without the presence of the domain wall. In this regime, the VHC is expected to be close to $e^{2} / h$. In fact, these measurements require less stringent conditions compared to those considered above, due to a wider energy range where the $\mathrm{VHC}$ is nonzero (as determined by the band splitting at the $K$ and $K^{\prime}$ points, resulting from the staggered sublattice potential) and the nonlocal voltage output being independent of the domain structure of $\mathrm{Cr}_{2} \mathrm{O}_{3}$.

\section{E. Topological phases across a domain wall}

The formation of the $180^{\circ}$ AFM domain wall also leads to a topological phase transition in graphene, resulting from continuous rotation of the surface magnetization. This transition manifests itself in the changing topological invariants across the domain wall. For the surface magnetization pointing normal to the interface, the QAHE is characterized by the Chern number $C=\frac{1}{2 \pi} \sum_{n} \int_{B Z} \Omega^{n}(\vec{k}) d^{2} k$ (where the summation is performed over all occupied bands) being +2 or -2 for $\theta=0^{\circ}$ and $\theta=180^{\circ}$, respectively. The Chern number is a topological invariant and thus must discontinuously change across the domain wall. At each discontinuity point, the transition must be accompanied by band-gap closure.

To observe this transformation, we use Hamiltonian (1) to calculate the evolution of the band structure as a function of magnetization angle $\theta$, assuming for simplicity that the magnetization is uniform. The band structures for different angles $\theta$ are depicted in Supplemental Figs. S4 and S5 [35] around the $K^{\prime}$ and $K$ points, respectively. We find that near the $K^{\prime}$ point the band gap closes at $\theta \approx 36^{\circ}$ [Fig. S4(b)], then it reopens [see Fig. $\mathrm{S} 4(\mathrm{c})$ for $\theta \approx 49^{\circ}$ ], closes again at $\theta \approx 60^{\circ}$ [Fig. S4(d)], and remains open at larger $\theta$ [Figs. S4(e)-S4(i)]. The band structure around the $K$ point mirrors that near the $K^{\prime}$ point when the magnetization is flipped from $\theta$ to $180^{\circ}-\theta$. In this case, the band gap is opened at smaller angles [Figs. S5(a)-S5(e)], but it closes at $\theta \approx 120^{\circ}$ [Fig. S5(f)], then it reopens [see Fig. S5(g) for $\theta \approx 131^{\circ}$ ], and closes again at $\theta \approx 144^{\circ}$ [Fig. S5(h)]. Between $36^{\circ}<\theta<60^{\circ}$ and $120^{\circ}<$ $\theta<144^{\circ}$, the maximum band opening is about $0.1 \mathrm{meV}$ at $\theta \approx 49^{\circ}$ (the $K^{\prime}$ point) and $\theta \approx 131^{\circ}$ (the $K$ point). These results indicate that multiple topological phases emerge in the range of angles $36^{\circ} \lesssim \theta \lesssim 144^{\circ}$.

To reveal the nature of these topological phases we calculate the valley-dependent Chern numbers, $C_{K}$ and $C_{K^{\prime}}$, by integrating the Berry curvature around the $K$ and $K^{\prime}$ points, respectively. In the calculation, we chose $\varepsilon_{f}$ to lie within the band gap for $\theta=0^{\circ}, \theta=49^{\circ}, \theta=90^{\circ}, \theta=131^{\circ}$, and $\theta=180^{\circ}$. We find that the valley-dependent Chern numbers are equal for the surface magnetization normal to the interface; i.e., $C_{K}=C_{K^{\prime}}=1$ for $\theta=0$ and $C_{K}=C_{K^{\prime}}=-1$ for $\theta=180^{\circ}$. These conditions produce a QAHE phase with the total Chern numbers $C=C_{K}+C_{K^{\prime}}= \pm 2$ and the valley Chern number $C_{v}=C_{K}-C_{K^{\prime}}=0$. However, for the other angles, we obtain that $C_{K}$ and $C_{K^{\prime}}$ are different, namely, $C_{K}=1$ and $C_{K^{\prime}}=0$ at $\theta=49^{\circ}, C_{K}=1$ and $C_{K^{\prime}}=-1$ at $\theta=90^{\circ}$, and $C_{K}=0$ and $C_{K^{\prime}}=-1$ at $\theta=131^{\circ}$, signaling for the emergence of different topological phases. The regions around $\theta=49^{\circ}$ and $\theta=131^{\circ}$ are characterized by $C= \pm 1$, respectively, and $C_{v}=1$. These regions exhibit the valley-polarized QAHE (VPQAHE) phase [46], where the QAHE and the VHE coexist. For magnetization lying in the plane, i.e., $\theta=90^{\circ}$, the resulting phase has zero total Chern number, $C=0$, but a nonzero valley Chern number, $C_{v}=2$, indicating the emergence of the quantum valley Hall effect (QVHE) [42]. The corresponding topological phase diagram is depicted in Fig. 6. We note that the overall situation is somewhat reminiscent of that predicted for a Bi bilayer where magnetization rotation induced by the spin-orbit torque forces the topological phase transition [36].

\section{DISCUSSION}

The emergence of different topological phases in graphene across the AFM domain wall in chromia is expected to produce chiral edge states (CESs) similar to those predicted [8] and observed [47-49] on domain walls of a magnetically 


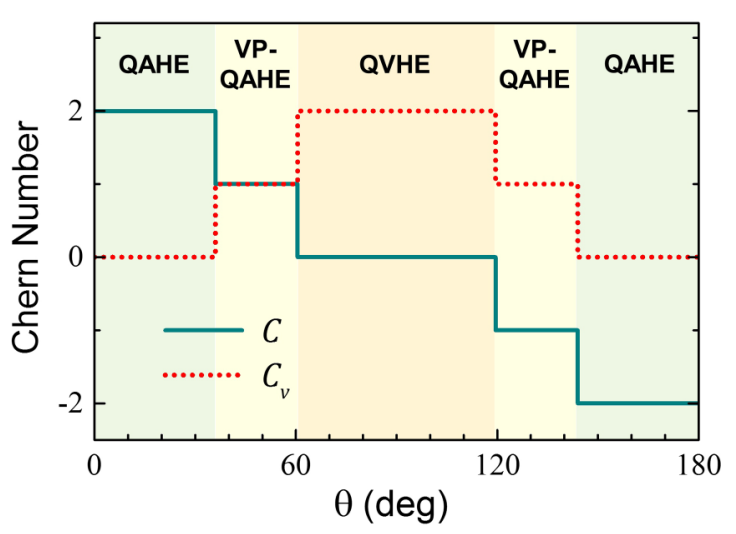

FIG. 6. Topological phase transformation as a function of magnetization angle $\theta$. Three topological phases are distinguished by the Chern numbers: QAHE $\left(C= \pm 2, C_{v}=0\right.$, indicated by green color); valley-polarized QAHE (VPQAHE) $\left(C= \pm 1, C_{v}=1\right.$, indicated by yellow color); and QVHE $\left(C=0, C_{v}=2\right.$, indicated by orange color).

doped topological insulator. CESs are expected to appear along the lines parallel to the domain wall where the topological phase changes. The appearance of the CESs, as well as the QVHE phase, requires a sufficiently wide domain wall with the width larger than the characteristic decay length of the CESs into the gapped region, which we estimate to be about $12 \mathrm{~nm}$ (Supplemental Material [35]). If the domain wall is not wide enough, the VP-QAHE and QVHE phases disappear.

Observation of the predicted phenomena relies on the magnetoelectricity of chromia. The latter allows a $180^{\circ}$ domain wall of the surface magnetization to be formed by applying voltages of different sign at two local regions to align the Néel vector in opposite directions [50]. The domain wall width can be engineered either by tuning anisotropy with strain or by the split-gate scheme with multiple gates. Furthermore, the locally applied voltages can dynamically control the location of the domain wall [50]. The dynamics may be used to switch paths of injected spin-polarized electrons, which can be detected for readout at specific probes locally attached to graphene along the voltage-controlled domain wall regions in chromia. A further advantage of the proposed scheme is that the interface with the split gates needs neither dopants, adatoms, nor external Oersted fields. The magnetoelectric switching is expected to improve the timing of the writing process as to form the domains in memory devices, since it is controlled by applied voltages, rather than by magnetic probe scanning [49]. Specific transport measurements to observe the predicted phenomena can be performed using a four-terminal probe [51-54]. The required $\varepsilon_{f}$ engineering can be achieved via applied gate voltage [55] or uniaxial pressure [34].

\section{CONCLUSIONS}

Our work has outlined a route for topological antiferromagnetic spintronics. We have predicted the control and manipulation of the topological states in a two-dimensional material via proximity of a magnetoelectric antiferromagnet with the Néel vector being the control parameter. Using the graphene $/ \mathrm{Cr}_{2} \mathrm{O}_{3}$ (0001) interface as a model system, we showed the emergence of the unconventional quantum anomalous Hall effect and the spin-polarized valley Hall effect. We predicted the appearance and transformation of the different topological phases in graphene across the $180^{\circ}$ AFM domain wall and the emergence of the chiral edge state along the domain wall. These topological properties can be controlled by voltage through magnetoelectric switching of the AFM insulator with no need for spin-orbit torques generated by large currents. Thus, our results provide a viable approach for low-power voltage-controlled topological antiferromagnetic spintronics.

\section{ACKNOWLEDGMENTS}

This work was supported by the National Science Foundation (NSF) through the E2CDA program (Grant No. ECCS-1740136) and the Semiconductor Research Corporation (SRC) through the nCORE program (Task No. 2760.001). A.A.K. and S.S. acknowledge support from the Department of Energy Early Career Award (Grant No. DE-SC0014189). Computations were performed at the University of Nebraska Holland Computing Center.

\section{APPENDIX: DFT COMPUTATIONAL DETAILS}

DFT calculations are performed using the Vienna $a b$ initio simulation package (VASP) [56]. We apply local density approximation (LDA) $+U$ [57] with $U=4.0 \mathrm{eV}$ and $J=0.58$ $\mathrm{eV}$ using the projector augmented-wave method [58]. In the calculations, we use a $\mathrm{Cr}_{2} \mathrm{O}_{3}$ (0001) slab composed of eight and 16 atomic layers of $\mathrm{O}$ and $\mathrm{Cr}$, respectively [Fig. 1(a)], assuming the energetically most favorable surface structure terminated with a single $\mathrm{Cr}$ layer top and bottom of the slab [59]. A $2 \times 2$ graphene sheet, whose lattice parameters are strained to the $\mathrm{Cr}_{2} \mathrm{O}_{3}$ (0001) lattice, is placed on the top [Fig. 1(b)] and bottom surfaces of the $\mathrm{Cr}_{2} \mathrm{O}_{3}$ slab. The in-plane placement of graphene on the $\mathrm{Cr}_{2} \mathrm{O}_{3}$ (0001) surface is optimized as described in the Supplemental Material [35]. Periodic boundary conditions along the $z$ direction are maintained with a 20 -Å-thick vacuum buffer layer separating the slabs. Under the constraint of the fixed in-plane lattice constant of $\mathrm{Cr}_{2} \mathrm{O}_{3}(a=4.94 \AA)$, the atomic structures are fully relaxed using the $9 \times 9 \times 1 \Gamma$-centered $k$-point grid and the kinetic energy cutoff of $520 \mathrm{eV}$. The relaxation is performed until the atomic forces on each atom are converged to better than $0.01 \mathrm{eV} / \AA$. In the band structure calculations, we increase the $k$-point grid up to $18 \times 18 \times 1$.
[1] Spintronics Handbook: Spin Transport and Magnetism, 2nd ed., edited by E. Y. Tsymbal and I. Žutić (CRC Press, Boca Raton, FL, 2019).

[2] A. H. MacDonald and M. Tsoi, Antiferromagnetic metal spintronics, Philos. Trans. R. Soc., A 369, 3098 (2011).
[3] T. Jungwirth, X. Marti, P. Wadley, and J. Wunderlich, Antiferromagnetic spintronics, Nat. Nanotechnol. 11, 231 (2016).

[4] V. Baltz, A. Manchon, M. Tsoi, T. Moriyama, T. Ono, and Y. Tserkovnyak, Antiferromagnetic spintronics, Rev. Mod. Phys. 90, 015005 (2018). 
[5] T. Jungwirth, J. Sinova, A. Manchon, X. Marti, J. Wunderlich, and C. Felser, The multiple directions of antiferromagnetic spintronics, Nat. Phys. 14, 200 (2018).

[6] B. Keimer and J. E. Moore, The physics of quantum materials, Nat. Phys. 13, 1045 (2017).

[7] A. H. Castro Neto, F. Guinea, N. M. R. Peres, K. S. Novoselov, and A. K. Geim, The electronic properties of graphene, Rev. Mod. Phys. 81, 109 (2009).

[8] M. Z. Hasan and C. L. Kane, Colloquium: Topological insulators, Rev. Mod. Phys. 82, 3045 (2010).

[9] N. P. Armitage, E. J. Mele, and A. Vishwanath, Weyl and Dirac semimetals in three-dimensional solids, Rev. Mod. Phys. 90, 015001 (2018).

[10] B. Bradlyn, J. Cano, Z. Wang, M. G. Vergniory, C. Felser, R. J. Cava, and B. A. Bernevig, Beyond Dirac and Weyl fermions: Unconventional quasiparticles in conventional crystals, Science 353, aaf5037 (2016).

[11] D. A. Pesin and A. H. MacDonald, Spintronics and pseudospintronics in graphene and topological insulators, Nat. Mater. 11, 409 (2012).

[12] Y. Fan, P. Upadhyaya, X. Kou, M. Lang, S. Takei, Z. Wang, J. Tang, L. He, L.-T. Chang, M. Montazeri, G. Yu, W. Jiang, T. Nie, R. N. Schwartz, Y. Tserkovnyak, and K. L. Wang, Magnetization switching through giant spin-orbit torque in a magnetically doped topological insulator heterostructure, Nat. Mater. 13, 699 (2014).

[13] A. R. Mellnik, J. S. Lee, A. Richardella, J. L. Grab, P. J. Mintun, M. H. Fischer, A. Vaezi, A. Manchon, E.-A. Kim, N. Samarth, and D. C. Ralph, Spin-transfer torque generated by a topological insulator, Nature 511, 449 (2014).

[14] L. Šmejkal, T. Jungwirth, and J. Sinova, Route towards Dirac and Weyl antiferromagnetic spintronics, Phys. Status. Solidi Rapid Res. Lett. 11, 1700044 (2017).

[15] L. Šmejkal, Y. Mokrousov, B. Yan, and A. H. MacDonald, Topological antiferromagnetic spintronics, Nat. Phys. 14, 242 (2018).

[16] L. Šmejkal, J. Železný, J. Sinova, and T. Jungwirth, Electric Control of Dirac Quasiparticles by Spin-Orbit Torque in an Antiferromagnet, Phys. Rev. Lett. 118, 106402 (2017).

[17] D.-F. Shao, G. Gurung, S.-H. Zhang, and E. Y. Tsymbal, Dirac Nodal Line Metal for Topological Antiferromagnetic Spintronics, Phys. Rev. Lett. 122, 077203 (2019).

[18] J. Železný, H. Gao, K. Výborný, J. Zemen, J. Mašek, A. Manchon, J. Wunderlich, J. Sinova, and T. Jungwirth, Relativistic Néel-Order Fields Induced by Electrical Current in Antiferromagnets, Phys. Rev. Lett. 113, 157201 (2014).

[19] P. Wadley, B. Howells, J. Železný, C. Andrews, V. Hills, R. P. Campion, V. Novák, K. Olejník, F. Maccherozzi, S. S. Dhesi, S. Y. Martin, T. Wagner, J. Wunderlich, F. Freimuth, Y. Mokrousov, J. Kuneš, J. S. Chauhan, M. J. Grzybowski, A. W. Rushforth, K. W. Edmonds et al., Electrical switching of an antiferromagnet, Science 351, 587 (2016).

[20] S. Y. Bodnar, L. Šmejkal, I. Turek, T. Jungwirth, O. Gomonay, J. Sinova, A. A. Sapozhnik, H.-J. Elmers, M. Kläui, and M. Jourdan, Writing and reading antiferromagnetic $\mathrm{Mn}_{2} \mathrm{Au}$ by Néel spin-orbit torques and large anisotropic magnetoresistance, Nat. Commun. 9, 348 (2018).

[21] X. He, Y. Wang, N. Wu, A. N. Caruso, E. Vescovo, K. D. Belashchenko, P. A. Dowben, and C. Binek, Robust isothermal electric control of exchange bias at room temperature, Nat. Mater. 9, 579 (2010).

[22] A. F. Andreev, Macroscopic magnetic fields of antiferromagnets, JETP Lett. 63, 758 (1996).

[23] K. D. Belashchenko, Equilibrium Magnetization at the Boundary of a Magnetoelectric Antiferromagnet, Phys. Rev. Lett. 105, 147204 (2010).

[24] N. Wu, X. He, A. L. Wysocki, U. Lanke, T. Komesu, K. D. Belashchenko, C. Binek, and P. A. Dowben, Imaging and Control of Surface Magnetization Domains in a Magnetoelectric Antiferromagnet, Phys. Rev. Lett. 106, 087202 (2011).

[25] S. Cao, A. Xiao, C.-P. Kwan, K. Zhang, J. P. Bird, L. Wang, W.-N. Mei, X. Hong, and P. A. Dowben, Moving towards the magnetoelectric graphene transistor, Appl. Phys. Lett. 111, 182402 (2017).

[26] F. D. M. Haldane, Model for a Quantum Hall Effect without Landau Levels: Condensed-Matter Realization of the "Parity Anomaly”, Phys. Rev. Lett. 61, 2015 (1988).

[27] C. L. Kane and E. J. Mele, Quantum Spin Hall Effect in Graphene, Phys. Rev. Lett. 95, 226801 (2005).

[28] Y. Yao, F. Ye, X.-L. Qi, S.-C. Zhang, and Z. Fang, Spin-orbit gap of graphene: First-principles calculations, Phys. Rev. B 75, 041401(R) (2007).

[29] M. Gmitra, S. Konschuh, C. Ertler, C. Ambrosch-Draxl, and J. Fabian, Band-structure topologies of graphene: Spin-orbit coupling effects from first principles, Phys. Rev. B 80, 235431 (2009).

[30] A. Avsar, J. Y. Tan, T. Taychatanapat, J. Balakrishnan, G. K. W. Koon, Y. Yeo, J. Lahiri, A. Carvalho, A. Rodin, E. C. T. O'Farrell, G. Eda, A. H. Castro Neto, and B. Özyilmaz, Spin-orbit proximity effect in graphene, Nat. Commun. 5, 4875 (2014).

[31] M. Gmitra and J. Fabian, Graphene on transition-metal dichalcogenides: A platform for proximity spin-orbit physics and optospintronics, Phys. Rev. B 92, 155403 (2015).

[32] H. X. Yang, A. Hallal, D. Terrade, X. Waintal, S. Roche, and M. Chshiev, Proximity Effects Induced in Graphene by Magnetic Insulators: First-Principles Calculations on Spin Filtering and Exchange-Splitting Gaps, Phys. Rev. Lett. 110, 046603 (2013).

[33] Z. Wang, C. Tang, R. Sachs, Y. Barlas, and J. Shi, ProximityInduced Ferromagnetism in Graphene Revealed by the Anomalous Hall Effect, Phys. Rev. Lett. 114, 016603 (2015).

[34] Z. Qiao, W. Ren, H. Chen, L. Bellaiche, Z. Zhang, A. H. MacDonald, and Q. Niu, Quantum Anomalous Hall Effect in Graphene Proximity Coupled to an Antiferromagnetic Insulator, Phys. Rev. Lett. 112, 116404 (2014).

[35] See Supplemental Material at http://link.aps.org/supplemental/ 10.1103/PhysRevB.100.125156 for comparisons of the relaxed interface structures with the different initial coordinates, the band structures for the lowest-energy interface structure and free-standing graphene, the parameters in the tight-binding Hamiltonian, and the band structures as a function of magnetization angles, and CES decay length.

[36] J.-P. Hanke, F. Freimuth, C. Niu, S. Blügel, and Y. Mokrousov, Mixed Weyl semimetals and low-dissipation magnetization control in insulators by spin-orbit torques, Nat. Commun. 8, 1479 (2017).

[37] W. Han, R. K. Kawakami, M. Gmitra, and J. Fabian, Graphene spintronics, Nat. Nanotechnol. 9, 794 (2014). 
[38] M. Gmitra, D. Kochan, and J. Fabian, Spin-Orbit Coupling in Hydrogenated Graphene, Phys. Rev. Lett. 110, 246602 (2013).

[39] T. P. Cysne, J. H. Garcia, A. R. Rocha, and T. G. Rappoport, Quantum Hall effect in graphene with interface-induced spinorbit coupling, Phys. Rev. B 97, 085413 (2018).

[40] T. Kosub, M. Kopte, R. Hühne, P. Appel, B. Shields, P. Maletinsky, R. Hübner, M. O. Liedke, J. Fassbender, O. G. Schmidt, and D. Makarov, Purely antiferromagnetic magnetoelectric random access memory, Nat. Commun. 8, 13985 (2017).

[41] A. Rycerz, J. Tworzydło, and C. W. J. Beenakker, Valley filter and valley valve in graphene, Nat. Phys. 3, 172 (2007).

[42] D. Xiao, W. Yao, and Q. Niu, Valley-Contrasting Physics in Graphene: Magnetic Moment and Topological Transport, Phys. Rev. Lett. 99, 236809 (2007).

[43] R. V. Gorbachev, J. C. W. Song, G. L. Yu, A. V. Kretinin, F. Withers, Y. Cao, A. Mishchenko, I. V. Grigorieva, K. S. Novoselov, L. S. Levitov, and A. K. Geim, Detecting topological currents in graphene superlattices, Science 346, 448 (2014).

[44] Y. D. Lensky, J. C. W. Song, P. Samutpraphoot, and L. S. Levitov, Topological Valley Currents in Gapped Dirac Materials, Phys. Rev. Lett. 114, 256601 (2015).

[45] T. Ando, Theory of valley Hall conductivity in graphene with gap, J. Phys. Soc. Jpn. 84, 114705 (2015).

[46] H. Pan, Z. Li, C. C. Liu, G. Zhu, Z. Qiao, and Y. Yao, Valley-Polarized Quantum Anomalous Hall Effect in Silicene, Phys. Rev. Lett. 112, 106802 (2014).

[47] J. G. Checkelsky, J. Ye, Y. Onose, Y. Iwasa, and Y. Tokura, Dirac-fermion-mediated ferromagnetism in a topological insulator, Nat. Phys. 8, 729 (2012).

[48] M. Liu, W. Wang, A. R. Richardella, A. Kandala, J. Li, A. Yazdani, N. Samarth, and N. P. Ong, Large discrete jumps observed in the transition between Chern states in a ferromagnetic topological insulator, Sci. Adv. 2, e1600167 (2016).

[49] K. Yasuda, M. Mogi, R. Yoshimi, A. Tsukazaki, K. Takahashi, S. S. Kawasaki, F. Kagawa, and Y. Tokura, Quantized chiral edge conduction on domain walls of a magnetic topological insulator, Science 358, 1311 (2017).

[50] K. D. Belashchenko, O. Tchernyshyov, A. A. Kovalev, and O. A. Tretiakov, Magnetoelectric domain wall dynamics and its implications for magnetoelectric memory, Appl. Phys. Lett. 108, 132403 (2016).

[51] R. Yu, W. Zhang, H.-J. Zhang, S.-C. Zhang, X. Dai, and Z. Fang, Quantized anomalous Hall effect in magnetic topological insulators, Science 329, 61 (2010).

[52] C.-Z. Chang, J. Zhang, X. Feng, J. Shen, Z. Zhang, M. Guo, K. Li, Y. Ou, P. Wei, L.-L. Wang, Z.-Q. Ji, Y. Feng, S. Ji, X. Chen, J. Ji, X. Dai, Z. Fang, S.-C. Zhang, K. He, Y. Wang et al., Experimental observation of the quantum anomalous Hall effect in a magnetic topological insulator, Science 340, 167 (2013).

[53] I. T. Rosen, E. J. Fox, X. Kou, L. Pan, K. L. Wang, and D. Goldhaber-Gordon, Chiral transport along magnetic domain walls in the quantum anomalous Hall effect, npj Quantum Mater. 2, 69 (2017).

[54] K. Komatsu, Y. Morita, E. Watanabe, D. Tsuya, K. Watanabe, T. Taniguchi, and S. Moriyama, Observation of the quantum valley Hall state in ballistic graphene superlattices, Sci. Adv. 4, eaaq0194 (2018).

[55] B. Huard, J. A. Sulpizio, N. Stander, K. Todd, B. Yang, and D. Goldhaber-Gordon, Transport Measurements Across a Tunable Potential Barrier in Graphene, Phys. Rev. Lett. 98, 236803 (2007).

[56] G. Kresse and J. Furthmuller, Efficient iterative schemes for ab initio total-energy calculations using a plane-wave basis set, Phys. Rev. B 54, 11169 (1996).

[57] A. I. Liechtenstein, V. I. Anisimov, and J. Zaanen, Densityfunctional theory and strong interactions: Orbital ordering in Mott-Hubbard insulators, Phys. Rev. B 52, 5467(R) (1995).

[58] P. E. Blöchl, Projector augmented-wave method, Phys. Rev. B 50, 17953 (1994).

[59] A. L. Wysocki, S. Shi, and K. D. Belashchenko, Microscopic origin of the structural phase transitions at the $\mathrm{Cr}_{2} \mathrm{O}_{3}(0001)$ surface, Phys. Rev. B 86, 165443 (2012). 